\title{
Effects of two-week e-learning on eHealth literacy: a randomized controlled trial of Japanese Internet users
}

\author{
Toshiharu Mitsuhashi ${ }^{\text {Corresp. } 1}$ \\ ${ }^{1}$ Center for Innovative Clinical Medicine, Okayama University, Okayama, Okayama prececture, Japan \\ Corresponding Author: Toshiharu Mitsuhashi \\ Email address: mitsuh-t@cc.okayama-u.ac.jp
}

Background. The Internet is widely used as a source of information by people searching for medical or healthcare information. However, information found on the Internet has several drawbacks, and the ability to consume accurate health information on the Internet (eHealth literacy) is increasingly important. This study's goal was to clarify the extent to which eHealth literacy is improved after e-learning in a randomized controlled trial.

Methods. Data were collected on 301 Japanese adults through an online survey.

Participants were assigned to the intervention (e-learning about eHealth literacy) group or the control group in a 1:1 ratio. The intervention group included 148 participants, and 153 participants were in the control group. The participants provided information at baseline on demographic characteristics, self-rated health, and frequency of Internet searching. The eHealth Literacy Scale (eHEALS), which was the main measure of eHealth literacy, and data on secondary outcomes (the Healthy Eating Literacy Scale and skill for evaluating retrieved search results) were obtained at baseline and at follow-up. The score difference was calculated by subtracting the score at baseline from the score at follow-up. Linear regression analysis and multinomial regression analysis were performed using the differences in score as the dependent variables and the intervention as the explanatory variable. Intention-to-treat analysis was employed. Results. The results from participants who responded to all of the questions both times were analyzed (134 in the intervention group and 148 in the control group). eHEALS increased 1.57 points due to the intervention effect ( $\Delta$ score change $=1.57 ; 95 \%$ Confidence Interval: $0.09,3.05 ; p=0.037$ ). Skills for evaluating retrieved search results improved more in the intervention group than in the control group (relative risk ratio $=2.47 ; 95 \%$ Confidence Interval: $1.33,4.59 ; p=0.004$ ) . There were no large differences at baseline between the intervention and control groups in the eHEALS, Healthy Eating Literacy scale, or skill for evaluating retrieved search results. However, at follow-up, the intervention group had improved more than the control group on both the eHEALS and skill for evaluating retrieved search results. Discussion. eHealth literacy improved after the e-learning, evidenced by the change to the eHEALS scores and 
increased skill for evaluating retrieved search results. There was no significant effect of elearning, which did not include content on healthy eating, on the Healthy Eating Literacy Scale scores. This indicates that scores did not increase much due to effects other than elearning, as is sometimes seen with the Hawthorne effect. Although it was statistically significant, the effect size was small. Therefore, future research is necessary to verify the clinical implications. In sum, this study suggests that e-learning is an effective way to improve eHealth literacy. 


\section{Effects of Two-week e-Learning on eHealth Literacy: A}

\section{Randomized Controlled Trial of Japanese Internet Users}

3 Toshiharu Mitsuhashi ${ }^{1}$

$4 \quad{ }^{1}$ Center for Innovative Clinical Medicine, Okayama University Hospital, Okayama, Japan

5

6 Corresponding Author:

7 Toshiharu Mitsuhashi ${ }^{1}$

8 2-5-1 Shikata-cho Kita-ku, Okayama, Okayama Prefecture, 700-8558, Japan

$9 \quad$ Email address: mitsuh-t@cc.okayama-u.ac.jp 
10

\section{Abstract}

Background. The Internet is widely used as a source of information by people searching for medical or healthcare information. However, information found on the Internet has several drawbacks, and the ability to consume accurate health information on the Internet (eHealth literacy) is increasingly important. This study's goal was to clarify the extent to which eHealth literacy is improved after e-learning in a randomized controlled trial.

Methods. Data were collected on 301 Japanese adults through an online survey. Participants were assigned to the intervention (e-learning about eHealth literacy) group or the control group in a 1:1 ratio. The intervention group included 148 participants, and 153 participants were in the control group. The participants provided information at baseline on demographic characteristics, self-rated health, and frequency of Internet searching. The eHealth Literacy Scale (eHEALS), which was the main measure of eHealth literacy, and data on secondary outcomes (the Healthy Eating Literacy Scale and skill for evaluating retrieved search results) were obtained at baseline and at follow-up. The score difference was calculated by subtracting the score at baseline from the score at follow-up. Linear regression analysis and multinomial regression analysis were performed using the differences in score as the dependent variables and the intervention as the explanatory variable. Intention-to-treat analysis was employed.

Results. The results from participants who responded to all of the questions both times were analyzed (134 in the intervention group and 148 in the control group). eHEALS increased 1.57 points due to the intervention effect $(\Delta$ score change $=1.57 ; 95 \%$ Confidence Interval: 0.09 , 3.05; $p=0.037$ ). Skills for evaluating retrieved search results improved more in the intervention group than in the control group (relative risk ratio $=2.47 ; 95 \%$ Confidence Interval: $1.33,4.59 ; p$ $=0.004)$. There were no large differences at baseline between the intervention and control groups in the eHEALS, Healthy Eating Literacy scale, or skill for evaluating retrieved search results. 
34 However, at follow-up, the intervention group had improved more than the control group on both

35 the eHEALS and skill for evaluating retrieved search results.

36 Discussion. eHealth literacy improved after the e-learning, evidenced by the change to the eHEALS scores and increased skill for evaluating retrieved search results. There was no significant effect of e-learning, which did not include content on healthy eating, on the Healthy

Eating Literacy Scale scores. This indicates that scores did not increase much due to effects other than e-learning, as is sometimes seen with the Hawthorne effect. Although it was statistically significant, the effect size was small. Therefore, future research is necessary to verify the clinical implications. In sum, this study suggests that e-learning is an effective way to improve eHealth literacy.

\section{Introduction}

The public widely uses the Internet as a source of medical and healthcare information. However, information found on the Internet has several drawbacks (Zhang, Sun \& Xie, 2015). First, available or retrieved information might be incomplete (De Groot et al., 2017; Takegami et al., 2017). Second, the information as written might not be clear (Daraz et al., 2018; De Groot et al., 2017). Third, even scientifically reliable information is not highly ranked in search engine results unless Search Engine Optimization is performed (Modave et al., 2014). Fourth, some problems with software tools that help users to organize and make sense of health information exist (Hernández et al., 2017). Fifth, the assessment tools of health information have important limitations (Beaunoyer et al., 2017). Therefore, scientifically reliable websites might not be retrieved, suggesting that information found on the Internet is not sufficient to obtain scientific reliability and reliance on it might actually be harmful to health (Bizzi, Ghezzi \& Paudyal, 2017; Kothari \& Moolani, 2015). 
Because of the unreliability of online information on health, it is important that people have the ability to critically appraise the health information that they obtain from the Internet. The skill involved with that ability is referred to as "health literacy," and is generally defined as "the ability to correctly examine and utilize health-related information" (Ad Hoc Committee on Health Literacy for the Council on Scientific Affairs, American Medical Association, 1999; Nutbeam, 1998; Sørensen et al., 2012). However, as evidenced by several surveys, the public's level of health literacy is not high. According to a German survey, 54.3\% of respondents were found to have limited health literacy (Schaeffer, Berens \& Vogt, 2017). In a survey from England, 52\% of respondents did not have an adequate score (Protheroe et al., 2017). According to a 2015 Japanese survey, about $85.4 \%$ of the respondents had health literacy problems (Nakayama et al., 2015). Thus, research indicates that health literacy is low on a global scale. However, Internet use rises every year, and it is increasingly important for the public to be able to obtain accurate information from the Internet for healthcare decision-making. Norman and Skinner (2006a) dubbed this ability "eHealth literacy" and defined it as "the ability to seek, find, understand, and appraise health information from electronic sources and apply the knowledge gained to addressing or solving a health problem" (p. 1). Since then, investigation of eHealth literacy has been limited, but survey results have found that people with low eHealth literacy might be likely to be exposed to incorrect or incomplete health information, which has been related to adverse health outcomes (De Boer, Versteegen \& van Wijhe, 2007). Therefore, education to improve eHealth literacy is important to public health.

Some previous studies have found that eHealth literacy improved after educational interventions (Robinson \& Graham, 2010; Xie, 2011a; Xie, 2011b). However, these studies had study design problems that interfered with the ability to demonstrate the effects of educational interventions. For example, a control group was not included and/or participants were not 
82 randomly assigned. Moreover, the influence of e-learning on eHealth literacy has not been studied. Therefore, this study aimed to clarify the extent to which eHealth literacy is influenced by e-learning in a randomized controlled trial in Japan.

\section{Materials \& Methods}

\subsection{Ethical considerations for studies on human subjects}

This study was approved by the Okayama University Graduate School of Medicine, Dentistry and Pharmaceutical Sciences and Okayama University Hospital, Ethics Committee (approval number K1707-025). The study was not registered because it does not meet the International Committee of Medical Journal Editors' criteria of a clinical experiment, and the study's results do not directly relate to patient outcomes. The purpose and method of research and experiment were appropriately described to potential participants on the recruitment webpage. After this description, informed consent was obtained from participants. They were free to refuse to participate for any reason.

\subsection{Trial design}

This study was a parallel, Internet-based, randomized controlled trial (RCT) of health literacy educational intervention by e-learning. First, a baseline questionnaire survey was administered online between September 29, 2017, and October 3, 2017. Then, the participants were 1:1 assigned to the intervention and control groups. The group receiving the treatment was exposed to e-learning for eHealth literacy during the 14 days from October 10, 2017, to October 23, 2017. A follow-up online questionnaire survey was administered from October 23, 2017 through October 30, 2017. This paper reports on the study using a modified Consolidated Standards of Reporting Trials (CONSORT) guideline checklist (www.consort-statement.org).

\subsubsection{Randomization}


After the baseline survey was completed, the participants were assigned to the intervention group or the control group using stratified block randomization with a block size of four in a 1:1 ratio. The participants were sorted into four strata by gender and age because both characteristics relate to eHealth literacy (Halwas, Griebel \& Huebner, 2017; Mitsutake et al., 2012). The participants were assigned to their groups by an automated system using Stata do-file mechanism, and, therefore, the investigator was not aware of, and did not personally participate in the group assignments. However, both groups could not be blinded.

\subsection{Participants}

This study's 300 participants were recruited from the population of about 1.2 million registered members of Macromill, Inc., which is a Japanese online survey company (https://monitor.macromill.com/). The participants were recruited from the member pool using four strata of 75 participants each: males aged 20 to 39 years, males aged 40 to 59 years, females aged 20 to 39 years, and females aged 40 to 59 years. The inclusion criteria were: (1) agreement to participate, (2) interest in e-learning, and (3) interest in health literacy. There were no exclusion criteria.

Recruitment was conducted from September 14 through 19, 2017. When the number of participants reached 300, recruitment was terminated. Because two participants simultaneously applied, the total sample size was 301. The sampling process is shown in Figure 1. Data on gender, age, residence, household income, and frequency of Internet search activity were collected in the baseline questionnaire. The participants were randomly assigned to the intervention $(n=148)$ or the control $(n=153)$ group after they completed the baseline questionnaire. Ultimately, 282 pieces of participant data were analyzed (134 in the intervention group and 148 in the control group) because 19 participants dropped out before the follow-up. 
130

131

132

133

134

135

136

137

138

139

140

141

142

143

144

145

146

147

148

149

150

151

152

153

\section{(Insert Fig. 1 here)}

32

All participants who answered every question were given 100 tokens (JPY 100, USD .94), and all of the participants who answered every question and completed the e-learning content were given 1,000 tokens (JPY 1,000, USD 9.36).

\subsubsection{Sample size calculation}

It was assumed that the primary outcome, eHealth Literacy Scale (eHEALS) scores of the intervention group, would improve by 2.0 points compared to the control group. In a previous study (Mitsutake et al., 2011), the standard deviation of eHEALS was 6.45. Because the participant data were considered similar to each other with respect to the inclusion criteria, the eHEALS scores were assumed to vary less and, therefore, the standard deviation was assumed to be smaller than previously found. It was expected to be about 6.0, and it was determined that $\alpha=$ 0.05 and $\beta=0.20$. Under these conditions, the required sample size was calculated as 143 per group. Considering sample attrition, the sample size was set at 150 per group.

\subsection{Trial intervention}

The intervention comprised e-learning content created by the researcher. Text material of elearning content has been prepared as a supplementary file. The content was presented to the participants in simple Japanese to facilitate comprehension. The content included text and images on the following topics: (1) reliability of information on the Internet, (2) scientific research methods, and (3) cautions regarding health information posted on social networking websites. The e-learning comprised 5,000 Japanese characters per topic. The entire e-learning content could easily be completed over a two-week period with about 10 minutes of dedicated application to learning the content per day. To confirm the participants' knowledge gained from the e-learning activity, four optional quizzes were included in the learning content. 


\subsection{Outcomes (dependent variables)}

All of the learning outcomes were measured using the participants' online responses to the baseline and follow-up questionnaires.

\subsubsection{Primary outcome (eHEALS)}

The eHEALS is an eight-item self-report questionnaire that assesses knowledge, comfort, and perceived skill at finding, evaluating, and applying electronic health information to health problems (Norman \& Skinner, 2006b). The response options on the items ranged from $1=$ not at all to $5=$ strongly agree. The responses on the items were summed, and these composite scores ranged from 8 to 40. The Japanese version of eHEALS was developed by Mitsutake et al. (2011). In the sample, Cronbach's alpha was 0.916 at baseline and 0.913 at follow up.

\subsubsection{Secondary outcomes}

This study assessed two secondary outcomes of e-learning: (1) the Healthy Eating Literacy scale (HEL), and (2) the skill for evaluating retrieved search results (evaluation skill).

The HEL is a five-item scale that measures interactive and critical literacy about healthy diet. The HEL was employed to assess change to health literacy other than change to eHealth literacy. The response options on the HEL's items ranged from $1=$ not at all to $5=$ strongly agree. Each subject was assigned a single score ranging from 1 to 5, which was the average of his or her responses on the five items. The HEL was developed by Kanae et al. (2012). Cronbach's alpha was 0.867 at baseline and 0.794 at follow up.

The HEL scale was used to examine the Hawthorne effect (Franke, 1978). Since the intervention group was observed more in detail, such as with the tracking of the number of elearning logins and the overall login time, than was the control group, the score might have risen due to the Hawthorne effect (McCarney et al., 2007). Since the intervention group did not learn about healthy eating through the e-learning content, the HEL score should not rise simply 
178 because of the e-learning. If that score did rise, it was considered to be evidence of the

179 Hawthorne effect.

180 The evaluation skill in this study was defined as the skill needed to evaluate the reliability of

181 webpages from retrieved search results with a limited amount of information. The participants'

182 evaluation skill was assessed using a question adapted from previous research (van Deursen \&

183 van Dijk, 2009; van Deursen \& van Dijk, 2010; van Deursen \& van Dijk, 2011). In a previous

184 study from 2011, the health literacy performance test was conducted using a laptop computer in a

185 university office to measure the four types of skills (operational, formal, information, and

186 strategic internet skills). However, since web questionnaires were conducted in this research, it

187 was difficult to measure operational, formal, and strategic internet skills. Therefore, in this study,

188 information skills were used to measure evaluation skills.

189 For assessment of evaluation skills, the participants were shown a results page with five

190 retrieved websites and asked to indicate which of the five websites should be viewed first. The

191 search results page, which was created for this study, listed two commercial websites, two

192 personal healthcare websites, and one governmental laboratory website. Search result summaries

193 and URL type (co.jp, com, ne.jp, and go.jp) were presented for the participants to use in

194 determining their choices. For the two commercial webpages, the URL types were co.jp and

195 com; from the title and the summary, it could be judged that the webpages were created by the

196 seller. For the two non-expert healthcare webpages, the URL types were co.jp and ne.jp, and

197 from the title and the summary, it could be judged that the webpages were created by non-

198 experts. The URL of the one governmental laboratory webpage was go.jp, and it was explicitly

199 stated that on this website, experts create articles for accurate information dissemination in the

200 results summary. The participants who selected the governmental laboratory were identified as

201 having mastered the evaluation skill. The participants with the skill were assigned one point, and 
202 those without the skill were assigned zero points. Change between the baseline and the follow-up

203 survey was computed by subtracting the baseline score from the follow-up scores. Calculation

204 results were $+1,0,-1$, which were defined as better, no change, and worse, respectively. This

205 measure has not been validated.

$206 \quad 2.6$ Statistical analysis

207 Participants who were in in the intervention group but did not learn the e-learning content

208 were analyzed as an intervention group (Intention-to-treat analysis). Statistical analysis was

209 performed using Stata (Stata Corporation, version 15, College Station, Texas, USA).

\section{$210 \quad$ 2.6.1 Descriptive statistics}

211 Means and standard deviations were used to describe the normally distributed continuous

212 variables, and medians and interquartile ranges were used to describe the non-normally

213 distributed continuous variables. Categorical variables were described using proportional

214 distributions.

\section{$215 \quad$ 2.6.2 Inferential statistics}

216 To estimate the influence of e-learning on eHEALS and HEL, differences between the scores

217 before and after (after scores minus before scores) the intervention were calculated. Linear

218 regression analyses were performed using the difference scores as the dependent variables and

219 the intervention as the explanatory variable, yielding unstandardized regression coefficients and

220 their 95\% confidence intervals (CIs). Next, Cohen's d and its 95\% CIs were calculated as the

221 effect size.

222 To estimate the influence of the intervention on evaluation skill, multinomial logistic

223 regression analysis was performed to regress the evaluation skill change on intervention, yielding

224 relative risk ratios (RRR) and their 95\% CIs using no change as the reference outcome (Hamilton,

225 1993). This model was selected since the dependent variable has more than two categories. The 
226 point estimate of RRR is calculated using the following equation.

227

$$
\begin{aligned}
R R R_{\text {outcome }} & =j \\
& =\frac{P(\text { outcome }=j \mid \text { intervention })}{P(\text { outcome }=\text { no change } \mid \text { intervention })} / \frac{P(\text { outcome }=j \mid \text { control })}{P(\text { outcome }=\text { no change } \mid \text { control })}
\end{aligned}
$$

For the significance test of the unstandardized regression coefficient, the Wald statistic and its 95\% CIs were calculated.

230

\subsubsection{Ancillary analysis}

Missing data on the dependent variables due to non-response at follow-up were handled

through multiple imputation by predictive means matching (Morris, White \& Royston, 2014).

The inferential analyses were performed on the complemented dataset $(n=301)$ as well as on the original dataset $(n=282)$.

Supplementarily, participants who were in in the intervention group but did not learn the elearning content were excluded from the analysis (per-protocol analysis).

\section{Results}

\subsection{Baseline characteristics}

Table 1 shows the participants' characteristics at baseline. The differences between the

intervention and control groups were small on most of the items. The proportion with university or more education was $54.7 \%$ in the intervention group and $63.4 \%$ in the control group. Selfrated health was $79.7 \%$ in the intervention group and $86.3 \%$ in the control group. Self-rated

245 health is a single-item summary measure of the perception of one's health. It is one suitable 246 method for measuring adult health status (Boardman, 2006). 
248 (Insert Table 1 here)

249

average, they completed $63.2 \%$ of the e-learning contents. Twenty-seven participants $(18.2 \%)$

did not even start the materials.

253

\subsection{Primary outcome (eHEALS)}

Table 2 shows the results regarding the eHEALS (means and standard deviations) and change between baseline and follow-up by group as well as differences between groups. There was a statistically significant difference between the intervention and control groups ( $\Delta$ score change $=1.57 ; 95 \% \mathrm{CI}=0.09,3.05 ; p=0.037)$

(Insert Table 2 here)

261

\subsection{Secondary outcomes}

Table 2 above shows the results regarding the HEL, which was not significantly different in the change between baseline and follow-up for either group (HEL: $\Delta$ score change $=-0.08 ; 95 \%$ $C I=-0.22,0.07 ; p=0.300)$. The proportional distribution of evaluation skill and its change after the intervention are displayed in Table 3. The intervention group was significantly likely to 267 change from "no change" to "better" $(\mathrm{RRR}=2.47 ; 95 \% \mathrm{CI}=1.33,4.59 ; p=0.004)$.

(Insert Table 3 here) 
271

272

273

274

275

276

277

278

279

280

281

282

283

284

285

286

\subsection{Results of the ancillary analysis}

Nineteen participants dropped out of the study before the follow-up survey. Fourteen dropped out of the intervention group and five dropped out of the control group. Their missing scores on the outcome change variables were estimated using multiple imputation. Table 4 shows the estimation results of the regression analysis performed on the complemented data set. This result was almost the same as the result using the original data set.

The results of per-protocol analysis are shown in the supplementary tables (Tables S1 and S2). The estimate of the learning effect was larger than the result of the intention to treat analysis, but it followed the same trend as the intention to treat analysis.

\section{(Insert Table 4 here)}

\section{Discussion}

The results of this study indicate that eHealth literacy improved after a two-week e-learning program. This improvement was found in the eHEALS scores and in the participants' skill in selecting appropriate websites from search results. However, there was no significant change in health literacy regarding the HEL.

These results support previous studies' findings. For example, Robinson and Graham (2010) found that, after a 50-minute educational treatment, the eHEALS' scores of 18 participants increased from 19 to 32 . Another previous study found that eHEALS' scores significantly increased in an elderly sample (assessed using Cohen's d) after an educational intervention (Xie, 2011a). In addition, the eHEALS scores in this sample significantly increased after intervention regardless of the educational or presentational method (Xie, 2011b). In the current study, the score improvement on eHEALS was not as large as in these previous studies, but the eHEALS 
295

296

297

298

299

300

301

302

303

304

305

306

307

308

309

310

311

312

313

scores increased by 2.31 points (standard deviation 7.27 ) after the intervention (Table 2).

Although the increased scores after educational intervention were consistent with previous studies, this study's effect sizes were relatively small. One reason for that inconsistency is that the learning effect on the e-learning platform might be weaker than the learning effect derived from other delivery methods. This possibility should be addressed by future research. Another reason for the difference might be that the participants did not learn sufficient content. In fact, 27 participants in the intervention group did not learn at all, and 10 participants learned only part of the content.

Furthermore, e-learning could be continuously employed after its content is prepared.

Therefore, when it has a sufficient learning effect, it is a cost-effective way to enhance health.

On the other hand, if the e-learning content were incorrect, it might be harmful, and, therefore, validation of content is important to the development of e-learning systems.

eHealth literacy is also influenced by differences in individual characteristics, such as age, educational attainment, healthcare experiences, Internet expertise, and so on (Mitsutake et al., 2012; Mitsutake et al., 2016; Park, Moon \& Baeg, 2014), and eHealth literacy might be influenced more by face-to-face education than by e-learning (Cox, Bowmer \& Ring, 2011; Robinson \& Graham, 2010; Xie, 2011a; Xie, 2011b). Thus, it is necessary to determine the types of learning environments and methods (or combinations thereof) that might enhance eHealth literacy across diverse backgrounds.

\subsection{Strengths}

This study has five important strengths. First, the randomized controlled trial demonstrated that e-learning is an effective way to educationally intervene because any causal inference would not be influenced by confounding bias. Second, the proportion of responses in the follow-up survey was very high (93.7\%), which minimized the effect of selection bias. Third, in the 
319 complemented dataset, eHEALS scores and evaluation skill increased due to the intervention.

320 This indicates there was little influence of dropouts. Fourth, not only the subjective score

321 (eHEALS), but also the objective score (evaluation skill), improved due to e-learning. Fifth, the

322 eHEALS rose significantly, but the HEL scale did not. This suggests that the increased scores

323 were scarcely influenced by the Hawthorne effect.

324

325

326

327

328

329

330

331

332

333

334

335

336

337

338

339

340

341

342

\subsection{Limitations}

Regarding the measures used in the analysis, the variables other than eHEALS and HEL were not validated, and the participants' evaluation skills might not have been correctly evaluated. However, the interpretation of the results was not distorted because statistically significant results were found on the primary outcome (eHEALS), which was validated. Using self-report data to assess outcomes might cause non-differential misclassification, but when this type of misclassification occurs, it does not influence the point estimates or widen the confidence intervals. Therefore, using self-report data in this study did not influence the interpretation of its results. Last, because the learning effect was evaluated after a short two-week period, it could not be determined whether the effect of e-learning was retained for a longer time. Follow-up studies that cover longer periods are recommended.

\subsection{Generalizability}

The results of this study have limited generalizability because it targeted participants with an existing interest in health literacy and e-learning. The tokens distributed to the participants might have encouraged the intervention group to be more motivated, and the e-learning participation rate was considered to be high. If the tokens had not been distributed, the participation rate would have been considered low in e-learning education for the general population. Therefore, elearning in the general population might yield a result different from that of this study.

\section{Conclusions}


Although this study has some weaknesses, its results using RCT could demonstrate that e-

344 learning education had a positive effect on eHealth literacy for Japanese Internet users.

345 Furthermore, this study suggests that e-learning is an effective way to improve eHealth literacy.

\section{Acknowledgments}

I am grateful to all of the study's participants for their participation and to Macromill, Inc. for implementing the online survey. I would like to thank Editage (www.editage.jp) for English language editing.

\section{References}

Ad Hoc Committee on Health Literacy for the Council on Scientific Affairs, American Medical Association. 1999. Health literacy: Report of the Council on Scientific Affairs. The Journal of the American Medical Association 281:552-557 DOI:10.1001/jama.281.6.552.

Beaunoyer E, Arsenault M, Lomanowska MA, Guitton MJ. 2017. Understanding online health information: Evaluation, tools, and strategies. Patient Education and Counseling 100:183-189 DOI:10.1016/j.pec.2016.08.028.

Bizzi I, Ghezzi P, Paudyal P. 2017. Health information quality of websites on periodontology. Journal of Clinical Periodontology 44:308-314 DOI: 10.1111/jcpe.12668.

Boardman JD. 2006. Self-rated health among U.S. adolescents. Journal of Adolescent Health 38:401-8 DOI:10.1016/j.jadohealth.2005.01.006.

Cox N, Bowmer C, Ring A. 2011. Health literacy and the provision of information to women with breast cancer. Clinical Oncology 23:223-227 DOI: 10.1016/j.clon.2010.11.010.

Daraz L, Morrow AS, Ponce OJ, Farah W, Katabi A, Majzoub A, Seisa MO, Benkhadra R, Alsawas M, Larry P, Murad MH. 2018. Readability of online health information: A meta- 
367

368

369

370

371

372

373

374

375

376

377

378

379

380

381

382 383

384

385

386

387

388

389

390

narrative systematic review. American Journal of Medical Quality Article first published online DOI: $10.1177 / 1062860617751639$.

De Boer MJ, Versteegen GJ, van Wijhe M. 2007. Patients' use of the Internet for pain-related medical information. Patient Education and Counseling 68:86-97 DOI: 10.1016/j.pec.2007.05.012.

De Groot L, Harris I, Regehr G, Tekian A, Ingledew P-A. 2017. Quality of online resources for pancreatic cancer patients. Journal of Cancer Education DOI: 10.1007/s13187-017-12908.

Franke RH, Kaul JD. 1978. The Hawthorne experiments: First statistical interpretation. American Sociological Review 43:623-643 DOI:10.2307/2094540

Halwas N, Griebel L, Huebner J. 2017. eHealth literacy, Internet and eHealth service usage: A survey among cancer patients and their relatives. Journal of Cancer Research and Clinical Oncology 143:2291-2299 DOI:10.1007/s00432-017-2475-6.

Hamilton LC. 1993. sqv8: Interpreting multinomial logistic regression. Stata Technical Bulletin $13: 24-28$.

Hernández MA, Sharit J, Pirolli P, Czaja SJ. 2017. Adapting information search tools for use by health consumers: Challenges and lessons for software designers. International Journal of Human-Computer Interaction 34:445-456 DOI: 10.1080/10447318.2017.1358546.

Kanae T, Kazuhiro H, Ai S, Toshio N, Yoshio N. 2012. Reliability and validity of the Healthy Eating Literacy Scale among Japanese adults: From online web research data. Japanese Journal of Health Education and Promotion 20:30-40 DOI: 10.11260/kenkokyoiku.20.30.

Kothari M, Moolani S. 2015. Reliability of "Google" for obtaining medical information. Indian Journal of Ophthalmology 63:267-9 DOI: 10.4103/0301-4738.156934.

McCarney R, Warner J, Iliffe S, van Haselen R, Griffin M, Fisher P. 2007. The Hawthorne 
391

392

393

394

395

396

397

398

399

400

401

402

403

404

405

406

407

408

409

410

411

412

413

414

Effect: a randomised, controlled trial. BMC Medical Research Methodology 7:30 DOI: 10.1186/1471-2288-7-30.

Mitsutake S, Shibata A, Ishii K, Oka K. 2012. Association of eHealth literacy with colorectal cancer knowledge and screening practice among Internet users in Japan. Journal of Medical Internet Research 14:e153 DOI: 10.2196/jmir.1927.

Mitsutake S, Shibata A, Ishii K, Oka K. 2016. Associations of eHealth literacy with health behavior among adult Internet users. Journal of Medical Internet Research 18:e192 DOI: 10.2196/jmir.5413.

Mitsutake S, Shibata A, Ishii K, Okazaki K, Oka K. 2011. Developing Japanese version of the eHealth Literacy Scale (eHEALS). Japanese Journal of Public Health 58:361-371 DOI: 10.11236/jph.58.5_361.

Modave F, Shokar NK, Peñaranda E, Nguyen N. 2014. Analysis of the accuracy of weight loss information search engine results on the Internet. American Journal of Public Health 104:1971-1978 DOI: 10.2105/AJPH.2014.302070.

Morris TP, White IR, Royston P. 2014. Tuning multiple imputation by predictive mean matching and local residual draws. BMC Medical Research Methodology 14:75 DOI: $10.1186 / 1471-2288-14-75$.

Nakayama K, Osaka W, Togari T, Ishikawa H, Yonekura Y, Sekido A, Matsumoto M. 2015. Comprehensive health literacy in Japan is lower than in Europe: A validated Japaneselanguage assessment of health literacy. BMC Public Health 15:505 DOI: 10.1186/s12889-015-1835-x.

Norman CD, Skinner HA. 2006a. eHealth literacy: Essential skills for consumer health in a networked world. Journal of Medical Internet Research 8:e9 DOI: 10.2196/jmir.8.2.e9.

Norman CD, Skinner HA. 2006b. eHEALS: The eHealth Literacy Scale. Journal of Medical 
Internet Research 8:e27 DOI: 10.2196/jmir.8.4.e27.

416 Nutbeam D. 1998. Health promotion glossary. Health Promotion International 13:349-364 DOI: 10.1093/heapro/13.4.349.

Park H, Moon M, Baeg JH. 2014. Association of eHealth literacy with cancer information seeking and prior experience with cancer screening. Computers, Informatics, Nursing 32:458-463 DOI: 10.1097/CIN.0000000000000077.

Protheroe J, Whittle R, Bartlam B, Estacio EV, Clark L, Kurth J. 2017. Health literacy, associated lifestyle and demographic factors in adult population of an English city: a cross-sectional survey. Health Expectations 20:112-119 DOI: 10.1111/hex.12440

Robinson C, Graham J. 2010. Perceived Internet health literacy of HIV-positive people through the provision of a computer and Internet health education intervention. Health Information \& Libraries Journal 27:295-303 DOI: 10.1111/j.1471-1842.2010.00898.x.

Schaeffer D, Berens EM, Vogt D. 2017. Health literacy in the German population: Results of a representative survey. Deutsches Ärzteblatt International 114: 53-60 DOI: 10.3238/arztebl.2017.0053.

Sørensen K, Van den Broucke S, Fullam J, Doyle G, Pelikan J, Slonska Z, Brand H. 2012. Health literacy and public health: A systematic review and integration of definitions and models. BMC Public Health 12:80 DOI: 10.1186/1471-2458-12-80.

Takegami Y, Seki T, Amano T, Higuchi Y, Komatsu D, Nishida Y, Ishiguro N. 2017. The poor quality and reliability of information on periacetabular osteotomy on the Internet in Japan. Nagoya Journal of Medical Science 79:375-385 DOI: 10.18999/nagjms.79.3.375.

van Deursen AJAM, van Dijk JAGM. 2009. Using the Internet: Skill related problems in users' online behavior. Interacting with Computers 21:393-402 DOI:

10.1016/j.intcom.2009.06.005. 
439 van Deursen AJAM, van Dijk JAGM. 2010. Measuring Internet skills. International Journal of

$440 \quad$ Human-Computer Interaction 26:891-916 DOI: 10.1080/10447318.2010.496338.

441 van Deursen AJAM, van Dijk JAGM. 2011. Internet skills performance tests: Are people ready 442 for eHealth? Journal of Medical Internet Research 13:e35 DOI: 10.2196/jmir.1581.

443 Xie B. 2011a. Older adults, e-health literacy, and collaborative learning: An experimental study. Journal of the American Society for Information Science and Technology 62:933-946 DOI: 10.1002/asi.21507.

Xie B. 2011b. Experimenting on the impact of learning methods and information presentation

Zhang Y, Sun Y, Xie B. 2015. Quality of Health Information for Consumers on the Web. Journal of the Association of Information Science and Technology 66: 2071-2084 DOI: 10.1002/asi.23311. 


\title{
Figure 1 (on next page)
}

\author{
Participant flowchart
}




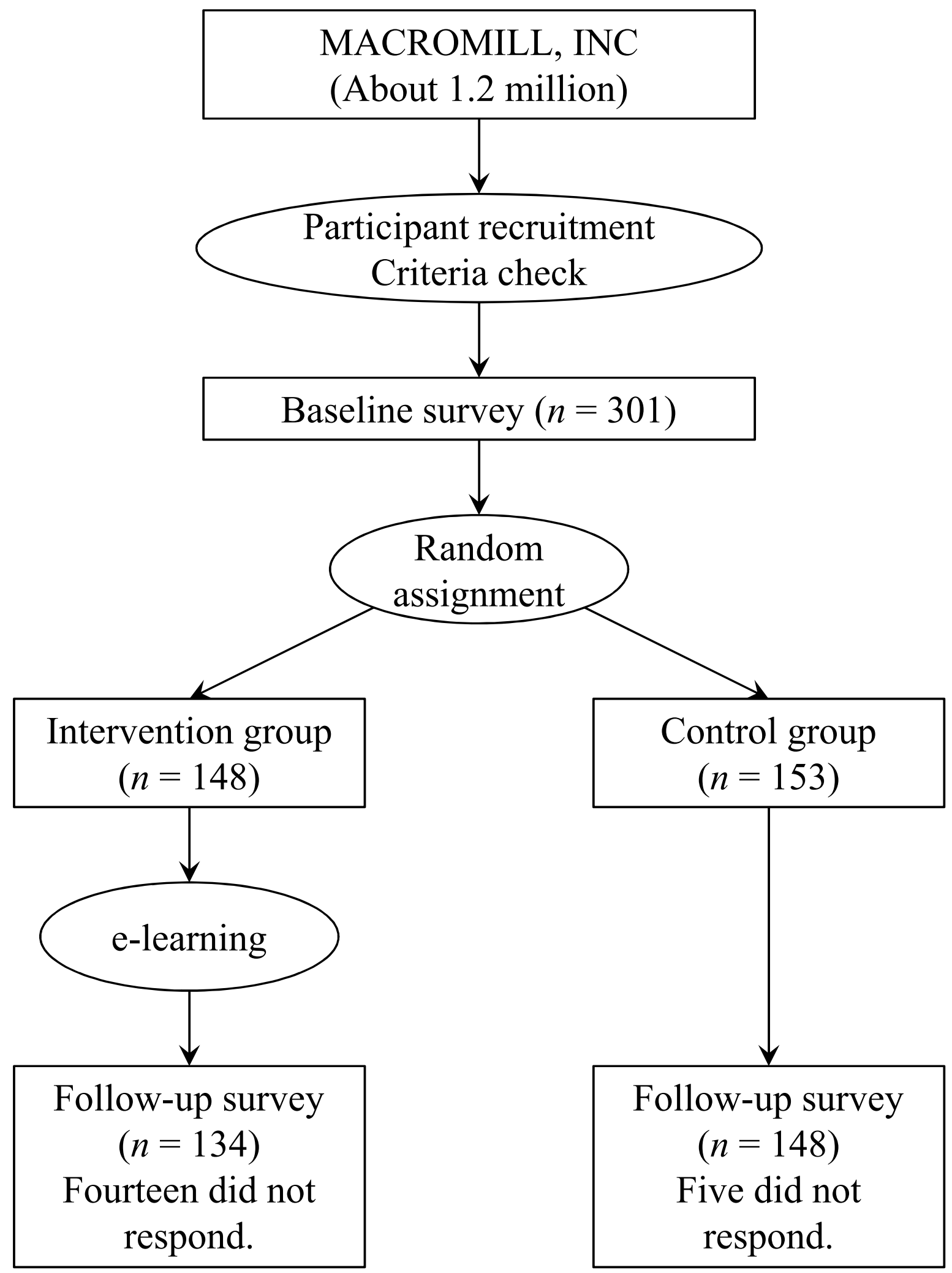


Table $\mathbf{1}$ (on next page)

Baseline characteristics of the sample 


\begin{tabular}{|c|c|c|c|}
\hline Variable & $\begin{array}{c}\text { Entire } \\
\text { sample } \\
(n=301)\end{array}$ & $\begin{array}{c}\text { Interventio } \\
\text { n group } \\
(n=148)\end{array}$ & $\begin{array}{c}\text { Control } \\
\text { group } \\
(n=153)\end{array}$ \\
\hline Female $(n(\%))$ & $150(49.8)$ & $74(50.0)$ & $76(49.7)$ \\
\hline Age in years (mean (SD)) & $40.2(10.1)$ & $40.2(9.9)$ & $40.2(10.2)$ \\
\hline Educational attainment of university or more $(n(\%))$ & $178(59.1)$ & $81(54.7)$ & $97(63.4)$ \\
\hline Parental status $(n(\%))$ & $133(44.2)$ & $67(45.3)$ & $66(43.1)$ \\
\hline \multicolumn{4}{|l|}{ Household income/year in JPY millions $(n(\%))$} \\
\hline Less than 2 & $19(6.3)$ & $10(6.8)$ & $9(5.9)$ \\
\hline 2 or more and less than 4 & $56(18.6)$ & $30(20.3)$ & $26(17.0)$ \\
\hline 4 or more and less than 6 & $78(25.9)$ & $35(23.6)$ & $43(28.1)$ \\
\hline 6 or more and less than 8 & $44(14.6)$ & $25(16.9)$ & $19(12.4)$ \\
\hline 8 or more and less than 10 & $22(7.3)$ & $12(8.1)$ & $10(6.5)$ \\
\hline 10 or more and less than 12 & $18(6.0)$ & $7(4.7)$ & $11(7.2)$ \\
\hline 12 or more and less than 15 & $3(1.0)$ & $1(0.7)$ & $2(1.3)$ \\
\hline 15 or more and less than 20 & $7(2.3)$ & $4(2.7)$ & $3(2.0)$ \\
\hline 20 or more & $3(1.0)$ & $1(0.7)$ & $2(1.3)$ \\
\hline Unknown & $16(5.3)$ & $9(6.1)$ & $7(4.6)$ \\
\hline Missing & $35(11.6)$ & $14(9.5)$ & $21(13.7)$ \\
\hline \multicolumn{4}{|l|}{ Marital status $(n(\%))$} \\
\hline Married & $161(53.5)$ & $82(55.4)$ & $79(51.6)$ \\
\hline Never married & $120(39.9)$ & $55(37.2)$ & $65(42.5)$ \\
\hline Divorced/widowed & $20(6.6)$ & $11(7.4)$ & $9(5.9)$ \\
\hline \multicolumn{4}{|l|}{ Employment status $(n(\%))$} \\
\hline Full-time & $163(54.2)$ & $73(49.3)$ & $90(58.8)$ \\
\hline Part-time & $46(15.3)$ & $29(19.6)$ & $17(11.1)$ \\
\hline Self-employed & $24(8.0)$ & $15(10.1)$ & $9(5.9)$ \\
\hline Other & $4(1.3)$ & $2(1.4)$ & $2(1.3)$ \\
\hline None & $64(21.3)$ & $29(19.6)$ & $35(22.9)$ \\
\hline Self-rated health $(n(\%))$ & $250(83.1)$ & $118(79.7)$ & $132(86.3)$ \\
\hline Internet search engine use $<$ once/day $(n(\%))$ & $43(14.3)$ & $19(12.8)$ & $24(15.7)$ \\
\hline
\end{tabular}




\section{Table 2 (on next page)}

Means, standard deviations (SD), change scores (follow-up minus baseline), and intervention effects ( $\Delta$ change) ${ }^{\text {a }}$ compared to control group 


\begin{tabular}{|c|c|c|c|c|}
\hline Dependent variable & Value & $\begin{array}{l}\text { Intervention } \\
\text { group } \\
(n=134)\end{array}$ & $\begin{array}{l}\text { Control } \\
\text { group } \\
(n=148)\end{array}$ & $\begin{array}{c}\text { Intervention v. } \\
\text { Control } \\
\Delta \text { change }^{\mathrm{a}} \text { and } \\
\text { Cohen's d (95\% } \\
\text { Confidence Interval) } \\
p \text {-value }\end{array}$ \\
\hline \multirow{3}{*}{$\begin{array}{l}\text { The eHealth Literacy } \\
\text { Scale, eHEALS (mean } \\
\text { (SD)) }\end{array}$} & Baseline & $24.5(6.61)$ & $25.9(6.18)$ & \multirow{3}{*}{$\begin{array}{c}1.57(0.09,3.05) \\
0.250(0.01,0.48) \\
p=0.037\end{array}$} \\
\hline & Follow-up & $26.8(5.84)$ & $26.6(5.63)$ & \\
\hline & Score change & $2.31(7.27)$ & $0.74(5.25)$ & \\
\hline \multirow{3}{*}{$\begin{array}{l}\text { The Healthy Eating } \\
\text { Literacy Scale, HEL } \\
\text { (mean (SD)) }\end{array}$} & Baseline & $3.44(0.71)$ & $3.52(0.70)$ & \multirow{3}{*}{$\begin{array}{c}-0.08(-0.22,0.07) \\
-0.12(-0.38,0.11) \\
p=0.300\end{array}$} \\
\hline & Follow-up & $3.50(0.63)$ & $3.65(0.54)$ & \\
\hline & Score change & $0.06(0.65)$ & $0.14(0.59)$ & \\
\hline
\end{tabular}

1 a Score change of intervention group minus score change of control group 


\section{Table 3(on next page)}

Results on evaluation skill at baseline and follow-up, intervention effect, and comparison of intervention group to control group 


\begin{tabular}{llccc}
\hline \multicolumn{1}{c}{ Variable } & Value & $\begin{array}{c}\text { Intervention } \\
\text { group } \\
(n=134)\end{array}$ & $\begin{array}{c}\text { Control } \\
\text { group } \\
(n=148)\end{array}$ & $\begin{array}{c}\text { Intervention v. } \\
\text { Control } \\
\text { Relative Risk Ratio (95\% } \\
\text { Confidence Interval) } \\
p \text {-value }\end{array}$ \\
\hline $\begin{array}{l}\text { Having evaluation skill } \\
(\mathrm{n}(\%))\end{array}$ & Baseline & $44(32.8)$ & $47(31.8)$ & \\
\hline & Follow-up & $70(52.2)$ & $46(31.1)$ & \\
$\begin{array}{l}\text { Change in evaluation skill } \\
(\mathrm{n}(\%))\end{array}$ & No change & $86(64.2)$ & $109(73.6)$ & $\begin{array}{c}\text { Reference outcome }) \\
0.70(0.32,1.53) \\
p=0.370\end{array}$ \\
\hline
\end{tabular}




\section{Table 4 (on next page)}

Intervention effect ( $\Delta$ change ${ }^{a}$ and Relative Risk Ratio) compared to control group using multiple imputation to create complemented dataset 
Results

Intervention v. Control
$\Delta$ change $^{\mathrm{a}}(95 \%$ Confidence Interval $)$

Score change on eHealth Literacy

Scale (eHEALS)

Score change on Healthy Eating Literacy scale (HEL)

Evaluation skill

$$
1.52(0.05,2.99)
$$

$-0.06(-0.21,0.08)$

Intervention v. Control

Relative Risk Ratio (95\% Confidence Interval)

\begin{tabular}{lcc}
\hline Better & $2.27(1.22,4.24)$ & 0.010 \\
No change & (Reference outcome) & \\
Worse & $0.72(0.33,1.58)$ & 0.414
\end{tabular}

1 a Score change of intervention group minus score change of control group 